\title{
Desempenho até a Desmama de Bezerros Nelore e Cruzas com Nelore ${ }^{1}$
}

\section{Antonio Carlos Cubas ${ }^{2}$, Daniel Perotto ${ }^{3}$, José Jorge dos Santos Abrahão ${ }^{4}$, Sílvio Carlos Mella ${ }^{5}$}

RESUMO - Foi analisado o desempenho ponderal pré-desmama de bezerros Nelore (N), Guzerá x N (GN), Red Angus x N (RN) e Marchigiana x N (MN), oriundos de um experimento de cruzamentos realizado na Estação Experimental do IAPAR de Paranavaí, nascidos no período de 1985 a 1998, produzidos por meio de inseminação artificial, em duas estações anuais de nascimento (janeiro a abril e julho a dezembro). Foi utilizado o método dos quadrados mínimos para análise de 721 observações de pesos ao nascimento (PNT) e 686 de pesos à desmama (PDS) e ganho médio diário de peso do nascimento até a desmama (GMD_ND). Para PNT, o efeito de classe de datas julianas com intervalos de dez dias entre classes mostrou-se mais útil para explicar variações de épocas de nascimento do que o mês de nascimento, cuja influência foi muito grande para PDS e GMD_ND. Para as três características avaliadas, houve importante efeito (linear e quadrático) da idade da vaca (dias) mãe do bezerro, bem como dos efeitos fixos de raça do touro ou grupo genético, ano de nascimento do bezerro e sexo do bezerro. A interação grupo genético x sexo do bezerro foi efeito importante para PNT, PDS e GMD_ND. O efeito aleatório de touro dentro de grupo genético foi importante para PNT, PDS e GMD_ND. As médias dos quadrados mínimos e respectivos erros-padrão, sempre na sequiência $\mathrm{N}, \mathrm{GN}, \mathrm{RN}$ e MN, foram: $28,5 \pm 0,38 \mathrm{~kg}, 29,0 \pm 0,44 \mathrm{~kg}, 29,4 \pm 0,46 \mathrm{~kg}$ e $31,3 \pm 0,47 \mathrm{~kg}$, para PNT; $141,3 \pm 1,47 \mathrm{~kg}, 147,6 \pm 1,61 \mathrm{~kg}, 167,5 \pm 1,72 \mathrm{~kg}$ e $162,0 \pm 1,89 \mathrm{~kg}$, para PDS; $0,510 \pm 0,007 \mathrm{~kg}, 0,540 \pm$ $0,008 \mathrm{~kg}, 0,627 \pm 0,008 \mathrm{~kg}$ e $0,583 \pm 0,009 \mathrm{~kg}$, para GMD_ND.

Palavras-chave: cruzamentos, gado de corte, peso pré-desmama

\section{Pre-weaning Performance of Purebred Nellore and Bos taurus $\mathbf{x}$ Nellore Crossbred Calves}

ABSTRACT - The pre-weaning performance of Nellore (N), Guzerá x N (GN), Red Angus x N (RN) and Marchigiana x N (MN) calves, from a crossbreeding experiment conducted at IAPAR Paranavaí Experimental Station, produced by artificial insemination, born between 1985 and 1998 within two birth seasons (January to April and July to December) was studied. The least squares method was utilized to analyze 721 observations of birth weight (PNT) and 686 of weaning weight (PDS) and average daily gain from birth to weaning (GMD_ND). For PNT, the effect of classes of Julian days with ten days interval between classes, showed to be more useful to explain birth seasons variations than the calf birth month, whose influence was very strong for PDS and GMD_ND. For the three evaluated traits, there was important effect (linear and quadratic) of age (days) of the dam of the calf, as a co-variable, as well as of the fixed effects of breed of sire or genetic group, calf birth year and calf sex. The genetic group x sex of calf interaction was significant for PNT, PDS and GMD_ND. The random effect of sire within genetic group was important for PNT, PDS and GMD_ND. The least squares means and respective standard errors, always in the sequence N, GN, RN and MN, were: $28.5 \pm 0.38 \mathrm{~kg}, 29.0 \pm 0.44 \mathrm{~kg}, 29.4 \pm 0.46 \mathrm{~kg}$ and $31.3 \pm 0.47$ for PNT; $141.3 \pm 1.47 \mathrm{~kg}, 147.6 \pm 1.61 \mathrm{~kg}, 167.5 \pm 1.72 \mathrm{~kg}$ and $162.0 \pm 1.89 \mathrm{~kg}$ for PDS; and $0.510 \pm 0.007 \mathrm{~kg}, 0.540 \pm$ $0.008 \mathrm{~kg}, 0.627 \pm 0.008$ and $0.583 \pm 0.009$ for GMD_ND.

Key Words: beef cattle, crossbreeding, growth traits, pre-weaning

\section{Introdução}

Fundamentalmente, existem dois métodos não mutuamente exclusivos que podem ser usados no melhoramento genético dos rebanhos: a seleção dentro de raças e o cruzamento entre raças. Tanto as diferenças dentro de raças quanto aquelas entre raças são importantes, razão pela qual a taxa de progresso genético pode ser maximizada pela combinação dos dois métodos.
Entretanto, ao contrário das diferenças genéticas dentro de raças, as diferenças entre raças, bem como a heterose e a complementariedade, fenômenos associados aos cruzamentos, podem ser explorados prontamente, dependendo apenas da caracterização das raças e de seus cruzamentos, a fim de se encontrar combinações de genótipos que melhor se ajustem às fases do processo produtivo, bem como às condições ambientes e exigências de mercado de cada

\footnotetext{
1 Projeto financiado pelo IAPAR/Programa Produção Animal.

2 Prof. Adjunto, curso de Agronomia UFMS, CEP 79804-970, Cx. Postal 533, Dourados/MS. E.mail: acubas@ceud.ufms.br

3 Pesquisador do IAPAR, Polo Regional de Curitiba, Cx. Postal 2301, CEP 80011-970, Curitiba/PR. E.mail: dperotto@pr.gov.br

4 Pesquisador do IAPAR, Est. Experimental de Paranavaí, CEP 87701-970, Cx. Postal 564, Paranavaí/PR. E.mail: cpviapar@celepar.gov.br

5 Engo-Agro., Rua Walter Hubacher 247-A, CEP 79750-000, Nova Andradina, MS.
} 
região. A utilização de cruzamentos entre raças geneticamente diferenciadas deve visar à exploração dos fenômenos da heterose (KOCH et al., 1985) e da complementariedade (CARTWRIGHT, 1970). Por meio dos cruzamentos, pode-se chegar à compatibilização entre o genótipo animal para peso e tamanho e as demandas da fase do processo produtivo emque ele será utilizado (GREGORY, 1961; CARTWRIGHT, 1970). Além disso, a resposta resultante da adoção de cruzamentos se faz notar muito mais rapidamente do que aquela oriunda da seleção (PRESTON e WILLIS, 1974).

A bovinocultura de corte caracteriza-se por duas fases distintas, que são a cria, segmento da atividade no qual o rebanho cresce em número, e a terminação, etapa em que o rebanho aumenta em peso. As características de maior importância na primeira fase são precocidade sexual, fertilidade, intervalo de partos, produção de leite, habilidade materna, rusticidade e tamanho adulto da vaca. Na etapa de terminação, têm maior importância velocidade de ganho de peso, eficiência alimentar e características que determinam o mérito da carcaça (CARTWRIGHT, 1970).

De acordo com CARTWRIGHT (1970), o aumento de velocidade de ganho de peso tem sido o objetivo explícito da maioria dos programas de melhoramento genético de bovinos de corte. No entanto, esta estratégia desloca a eficiência do processo produtivo para a etapa de terminação, podendo resultar em perda de eficiência na fase de cria, pois implica na manutenção de vacas mais pesadas, além de aumentar em muito o risco de prejuízos causados por distocias e natimortos (CUBAS et al., 1991; BERGER et al., 1992). Esse fato é conseqüência do conflito de interesses entre as duas fases da produção e também da correlação genética positiva entre velocidade de ganho de peso no período de crescimento e peso à maturidade (FITZHUGH e TAYLOR, 1971). Em síntese, para o pecuarista brasileiro que se dedica à terminação, são interessantes programas de cruzamentos do gado Nelore com raças continentais pesadas (francesas, italianas, alemãs), ao passo que, para o criador, seriam mais importantes programas de cruzamentos entre o Nelore e raças britânicas de corte. Cruzamentos com raças britânicas, além de garantirem ao criador novilhas mestiças pesando pouco mais que as Nelore, asseguram também a exacerbação das características de eficiência reprodutiva e de habilidade materna.

Este artigo resulta de dados coletados em um experimento de cruzamentos entre raças bovinas de corte, tendo como base matrizes da raça Nelore, com o objetivo de oferecer aos produtores resultados alternativos à produção de carne exclusiva com animais Nelore, visando a exploração de possíveis benefícios oriundos da heterose e complementariedade entre as raças.

\section{Material e Métodos}

Foram analisadas 721 observações de pesos ao nascimento (PNT) e 686 de pesos à desmama (PDS) e do ganho médio diário de peso do nascimento até a desmama (GMD_ND) de bezerros Nelore (N), $1 / 2$ Guzerá $+1 / 2$ Nelore (GN), $1 / 2$ Red Angus $+1 / 2$ Nelore (RN) e $1 / 2$ Marchigiana $+1 / 2$ Nelore $(M N)$, machos e fêmeas, nascidos no período de 1985 a 1998, oriundos de um experimento planejado de cruzamentos de animais da raça Nelore, realizado na Estação Experimental do IAPAR de Paranavaí, Região Noroeste do Paraná, cujo clima é enquadrado no tipo Cfa da classificação de Köeppen (IAPAR, 1974), com solos dos grupos Latosol Vermelho Amarelo (MINISTÉRIO DA AGRICULTURA, 1970). Para PNT, a amostra continha dados de 240 bezerros N, 159 GN, $177 \mathrm{RN}$ e $145 \mathrm{MN}$ e, para PDS e GMD_ND, 224 N, 155 GN, 171 RN e 136 MN.

Os bezerros, nascidos no período de 14 anos, foram gerados via inseminação artificial, com sêmen de 97 touros (40 Nelore, 19 Guzerá, 17 Red Angus e 21 Marchigiana), adquirido de maneira aleatória, de acordo com a disponibilidade de sêmen de cada raça envolvida no programa, nas centrais de inseminação artificial a cada ano, com a garantia de participação de pelo menos cinco touros de cada raça por ano, com pelo menos um touro sendo repetido, para garantia da conectabilidade dos dados gerados. Duas estações de inseminação foram utilizadas a cada ano, de $1 \underline{\mathrm{O}}$ de outubro a 30 de janeiro e de 1 o de abril a 30 de junho. As vacas foram mantidas em pastagens de brachiaria (Brachiaria humidicula e Brachiaria decumbens), com suplementação no período seco via fornecimento de capim-napier (Pennisetum purpureum) e canade-açúcar (Saccharum officinarum) picados, pastejo direto em bancos de proteínas (Cajanus indicus e Leucaena leucocephala), além de uréia adicionada à mistura mineral. Os bezerros foram desmamados aproximadamente aos sete meses de idade. O rebanho foi submetido a rigoroso manejo sanitário, incluindo desverminações e pulverizações em épocas estratégicas para o controle de endo e ectoparasitoses, bem como vacinações sistemáticas contra febre aftosa, brucelose, carbúnculo hemático e paratifo dos bezerros. As variáveis peso à desmama e ganho médio diário de 
peso do nascimento à desmama foram submetidas a ajuste prévio para a idade de 210 dias, por meio de coeficientes de regressão sobre a idade, estimados dentro de grupo genético, ano de nascimento e sexo do bezerro.

As observações de cada característica foram analisadas pelo método dos quadrados mínimos (SAS, 1985), de acordo com o seguinte modelo linear :

$\mathrm{Y}_{\mathrm{ijk} l m n}=\mu+\mathrm{G}_{\mathrm{i}}+\mathrm{T}(\mathrm{G}) \mathrm{i}_{\mathrm{j}}+\mathrm{S}_{\mathrm{k}}+\mathrm{M}_{\mathrm{l}}+\mathrm{A}_{\mathrm{m}}+\mathrm{GS}_{\mathrm{ik}}+\mathrm{E}_{\mathrm{ijklmn}}$ em que $Y_{i j k l m n}=$ Valor observado para a característica (PNT, PDS e GMD_ND) no n-ésimo animal, nascido no m-ésimo ano e l-ésimo mês, do k-ésimo sexo, progênie do j-ésimo touro, dentro do i-ésimo grupo genético (erro para testar $\mathrm{G}$ ); $\mu=$ média geral da característica $Y ; \mathrm{G}_{\mathrm{i}}=$ efeito fixo do i-ésimo grupo genético $(\mathrm{i}=1,2,3,4) ; \mathrm{T}(\mathrm{G}) \mathrm{i}_{\mathrm{j}}=$ efeito aleatório do $\mathrm{j}$-ésimo touro $(\mathrm{j}=1,2,3, \ldots, \mathrm{n})$, dentro do i-ésimo grupo genético; $\mathrm{S}_{\mathrm{k}}=$ efeito fixo do k-ésimo sexo do bezerro ( $k=1$, macho e 2, fêmea); $M_{1}=$ efeito fixo do 1-ésimo mês de nascimento do bezerro $(1=1,2,3,4$, $7, \ldots, 12) ; \mathrm{A}_{\mathrm{m}}=$ efeito fixo do m-ésimo ano de nascimento do bezerro $(\mathrm{m}=1985, \ldots, 1998)$; $\mathrm{GS}_{\mathrm{ik}}=$ efeito da interação entre o i-ésimo grupo genético e o k-ésimo sexo do bezerro; e
$\mathrm{E}_{\mathrm{ijklmn}}=$ erro aleatório associado a cada observação.

Para a característica PNT, o mês de nascimento não foi efeito importante, sendo substituído no modelo pelo efeito de classes julianas de nascimento (CAMPOS et al., 1989), geradas com intervalos de dez dias entre classes, como covariável. Para as características PDS e GMD_ND, as classes julianas de nascimento não se mostraram importantes, sendo retiradas dos modelos de análise. A idade da vaca em anos, como covariável, teve importância para as três características, como efeitos linear e quadrático, sendo mantida nos modelos. O uso de polinômios segmentados (GALLANT e FULLER, 1973), para o fator idade da vaca, não melhorou o coeficiente de determinação das análises e não foi importante para qualquer das três características avaliadas, sendo abandonado.

\section{Resultados e Discussão}

O resumo das análises de variância das três características avaliadas pode ser verificado na Tabela 1. A geração de classes de datas julianas de nascimento, com intervalos de dez dias entre classes,

Tabela 1 - Resumo das análises de variância de características ponderais em bovinos Nelore e cruzas com Nelore, em Paranavaí-PR

Table 1 - Summary of the analyses of variance of growth traits in crossbred and purebred Nellore cattle, in Paranavaí-PR

\begin{tabular}{|c|c|c|c|c|}
\hline \multirow[t]{2}{*}{$\begin{array}{l}\text { Fonte de variação } \\
\text { Source of variation }\end{array}$} & \multirow[t]{2}{*}{$\begin{array}{l}\mathrm{gl} \mathrm{l}^{1} \\
d f^{1}\end{array}$} & \multicolumn{3}{|c|}{$\begin{array}{l}\text { Valor do F computado } \\
\text { Computed F value }\end{array}$} \\
\hline & & $\mathrm{PNT}^{2}$ & $\mathrm{PDS}^{2}$ & GMD_ND 2 \\
\hline $\begin{array}{l}\text { Classes de datas julianas } \\
\text { Julian days classes }\end{array}$ & 1 & $8,17 * * *$ & - & - \\
\hline $\begin{array}{l}\text { Raça ou grupo genético } \\
\text { Genetic group or breed }\end{array}$ & 3 & $7,55 * * *$ & $57,80 * * *$ & $46,29 * * *$ \\
\hline $\begin{array}{l}\text { Touro (Grupo genético) } \\
\text { Sire (Genetic group) }\end{array}$ & 93 & $1,34 *$ & $1,47 * * *$ & $1,38 * *$ \\
\hline $\begin{array}{l}\text { Sexo do bezerro } \\
\text { Calf sex }\end{array}$ & 1 & $18,94 * * *$ & $84,46 * * *$ & $52,10 * * *$ \\
\hline $\begin{array}{l}\text { Grupo genético x Sexo bezerro } \\
\text { Genetic group x Calf sex }\end{array}$ & 1 & $6,51 * * *$ & $5,55 * * *$ & $3,63 * *$ \\
\hline $\begin{array}{l}\text { Ano de nascimento do bezerro } \\
\text { Year of calf birth }\end{array}$ & 13 & $2,32 * * *$ & $27,14 * * *$ & $16,75^{* * *}$ \\
\hline $\begin{array}{l}\text { Mês de nascimento do bezerro } \\
\text { Month of calf birth }\end{array}$ & 9 & - & $7,57 * * *$ & $5,75 * * *$ \\
\hline $\begin{array}{l}\mathrm{IDM}(\mathrm{dia} / \text { day })^{3} \\
I D M Q(\text { dia/day })^{3} \\
\mathrm{R} \text { ao quadrado } \\
R \text { square }\end{array}$ & $\begin{array}{l}1 \\
1\end{array}$ & $\begin{array}{l}5,94 * * * \\
7,42 * * * \\
0,35\end{array}$ & $\begin{array}{l}12,68 * * * \\
15,08 * * * \\
0,71\end{array}$ & $\begin{array}{l}12,90 * * * \\
13,00 * * * \\
0,66\end{array}$ \\
\hline $\mathrm{CV}(\%)$ & & 11,40 & 8,41 & 10,67 \\
\hline
\end{tabular}


embora não tenha sido importante para PDS e GMD_ND, teve grande importância $(\mathrm{P}<0,01)$ para PNT. Como o mês de nascimento não foi importante para PNT, é razoável a suposição de que, sendo os intervalos de 30 dias muito longos e as diferenças entre os PNT relativamente pequenas, as classes de datas julianas, com intervalos de apenas dez dias, tenham sido mais eficazes na captação deste efeito ambiental sobre PNT, uma vez que o desenvolvimento fetal é linearmente intensificado nos últimos 60 dias que antecedem o parto (KOLB, 1984). Já para o PDS e o GMD_ND, o efeito do mês de nascimento dos bezerros foi significativo $(\mathrm{P}<0,01)$, pois as diferenças de qualidade dos pastos nos diferentes meses têm forte impacto na produção de leite das vacas, causando variações no ritmo de crescimento dos bezerros.

Os bezerros nascidos nos meses de julho, agosto e setembro tiveram os maiores GMD_ND $(\mathrm{P}<0,05)$ e PDS $(\mathrm{P}<0,01)$ em relação aos animais nascidos nos demais meses de nascimento. Os animais nascidos em julho tiveram maiores PDS $(\mathrm{P}<0,01)$ que os nascidos em agosto, os quais foram mais pesados $(\mathrm{P}<0,01)$ do que os bezerros de setembro, ou seja, a partir dos nascimentos de julho, ocorre tendência de queda linear do PDS. Não houve diferenças relevantes entre os meses de nascimento em que os bezerros tiveram os piores desempenhos, para ambas as características citadas.

A superioridade no desempenho pré-desmama e à desmama dos bezerros nascidos no início da estação de nascimentos de primavera/verão é bastante conhecida, embora não se encontrem na literatura explicações biológicas para o fenômeno. Possivelmente este melhor desempenho possa ser explicado por se tratar de um período que, embora pobre em oferta de forragens, é aquele que imediatamente antecede a época de melhor qualidade das pastagens. Como os bezerros têm sua melhor fase de velocidade de crescimento pré-desmama após os 60 dias de vida, quando conseguem ingerir toda a oferta de leite materno e passam a se comportar como ruminantes e começam a pastar, são duplamente beneficiados com o período de melhor qualidade e produção forrageira (outubro a dezembro). Conseqüentemente, se o pecuarista desejar usufruir deste fator para maximização do desempenho pré-desmama de seus animais, deve iniciar a estação de monta no mês de setembro (mais tardar início de outubro) de cada ano.

Ano de nascimento do bezerro explica muito $(\mathrm{P}<0,01)$ das diferenças de desenvolvimento verificadas nas três características avaliadas, indicando grande variação dos fatores edafo-climáticos entre anos. A idade da mãe em dias também foi importante fonte de variação no desempenho das crias, tanto como efeito linear quanto como efeito quadrático $(\mathrm{P}<0,01)$, indicativo de que tanto fêmeas jovens quanto matrizes velhas tendem a produzir bezerros mais leves. Importantes efeitos fixos de ano e mês de nascimento do bezerro, idade ou ordem de parto da vaca e sexo do bezerro têm sido relatados (ALENCAR et al., 1995; ALENCAR et al., 1997; ALENCAR et al., 1998; EUCLIDES FILHO et al., 1998; PEROTTO et al., 1998).

Detectou-se importante dimorfismo sexual $(\mathrm{P}<0,01)$, com os machos sempre pesando mais que as fêmeas (com exceção de PNT no grupo MN, o qual foi mais pesado nas fêmeas). Os valores que atestam a superioridade dos machos sobre as fêmeas já foram relatados previamente (PEROTTO et al., 1999). O efeito aleatório de touro dentro de cada grupo genético foi significativo para PNT $(\mathrm{P}<0,05)$, PDS $(\mathrm{P}<0,01)$ e GMD_ND $(\mathrm{P}<0,01)$, indicativo de marcantes diferenças entre touros dentro de cada raça. Importantes diferenças entre raças de touros $(\mathrm{P}<0,01)$ ocorreram e podem ser melhor visualizadas

Tabela 2 - Médias dos quadrados mínimos e respectivos erros-padrão de características ponderais em bovinos Nelore e cruzas com Nelore, em Paranavaí-PR

Table 2 - Least squares means and respective standard errors of growth traits in crossbred and purebred Nellore cattle, in Paranavaí-PR

\begin{tabular}{lcrr}
\hline $\begin{array}{l}\text { Grupo genético } \\
\text { Genetic group }\end{array}$ & \multicolumn{3}{c}{$\begin{array}{c}\text { Médias dos quadrados mínimos e erros-padrão (kg) } \\
\text { Least square means and standard errors }\end{array}$} \\
\cline { 2 - 4 } & PNT $^{1}$ & PDS $^{1}$ & GMD_ND $^{1}$ \\
\hline Nelore & $28,5 \pm 0,38$ & $141,3 \pm 1,47$ & $0,510 \pm 0,007$ \\
Guzerá x Nelore & $29,0 \pm 0,44$ & $147,6 \pm 1,61$ & $0,540 \pm 0,008$ \\
Red Angus x Nelore & $29,4 \pm 0,46$ & $167,5 \pm 1,72$ & $0,627 \pm 0,008$ \\
Marchigiana x Nelore & $31,3 \pm 0,47$ & $162,0 \pm 1,89$ & $0,583 \pm 0,009$
\end{tabular}

${ }_{1}^{1}$ PNT = peso ao nascimento; PDS = peso à desmama; GMD_ND = ganho médio diário de peso do nascimento à desmama.

${ }^{1} P N T=$ calf birth weight; $P D S=$ calf weaning weight; $G M D \_N D=$ average daily gain from birth to weaning . 
na Tabela 2, em que são apresentadas as médias e respectivos erros-padrão das características avaliadas para cada grupo genético.

Para PNT, não foram detectadas diferenças entre os pesos de animais dos grupos N, GN e RN, embora os bezerros $\mathrm{MN}$ tenham nascido significativamente $(\mathrm{P}<0,01)$ mais pesados do que aqueles dos demais grupos. Já para o PDS, foram marcantes as diferenças entre todos os possíveis pares de grupos genéticos $(\mathrm{P}<0,01)$, sendo a única diferença menos expressiva $(\mathrm{P}<0,05)$ aquela verificada entre bezerros RN e MN. Para GMD_ND, todas as diferenças entre grupos genéticos foram altamente relevantes $(\mathrm{P}<0,01)$, sendo os maiores ganhos diários, respectivamente, os dos bezerros RN, MN, GN e Nelore. Diferenças entre grupos genéticos em trabalhos de cruzamento com base em rebanhos Nelore, detectando maiores pesos e ganhos de peso nos animais cruzados têm sido reportados (ALENCAR et al., 1995; EUCLIDES FILHO et al., 1996; ALENCAR et al., 1997; ALENCAR et al., 1998; EUCLIDES FILHO et al., 1998; MUNIZ e QUEIROZ, 1998).

As médias dos quadrados mínimos e respectivos erros-padrão de PNT, PDS e GMD_ND, para cada sexo e grupo genético, são apresentadas na Tabela 3. Os bezerros Nelore e GN machos foram, respectivamente, 2,6 e 2,2 kg mais pesados ao nascer, desmamaram com 15,2 e 12,8 kg a mais e ganharam 55 e $52 \mathrm{~g}$ a mais por dia, no período pré-desmama, do que as fêmeas dos mesmos grupos genéticos, valores altamente significativos $(\mathrm{P}<0,01)$. A diferença de $0,6 \mathrm{~kg}$ ao nascimento entre machos e fêmeas $\mathrm{RN}$ não foi importante, mas sim a superioridade de $9,2 \mathrm{~kg}$ à desmama e de $36 \mathrm{~g}$ de ganho médio diário $(\mathrm{P}<0,01)$. Já as fêmeas MN foram $0,5 \mathrm{~kg}$ mais pesadas que os machos, embora tenham pesado $3,1 \mathrm{~kg}$ a menos na desmama e ganho $9 \mathrm{~g}$ a menos por dia que antecedeu a desmama, nenhuma destas diferenças sendo relevantes. Este comportamento diferenciado de dimorfismo sexual entre machos e fêmeas justifica as interações altamente significativas $(\mathrm{P}<0,01)$ entre grupo genético e sexo do bezerro para PNT e PDS, interação que foi importante $(\mathrm{P}<0,05)$ também para GMD_ND, conforme pode ser melhor apreciado nas Figuras 1, 2 e 3.

Interações significativas entre grupo genético e sexo do bezerro encontram concordância, embora sem que tenha sido detectada a inversão de valores (como no caso de PNT do grupo MN), mas sim falta de uniformidade entre as magnitudes das diferenças entre os sexos nos diferentes grupos (MAZZA, 1984; EUCLIDES FILHO et al., 1996). De acordo com MAZZA (1984), uma possível explicação biológica para a interação grupo genético x sexo do bezerro é o antagonismo entre o potencial de crescimento do bezerro e o ambiente intra-uterino materno, ou seja, o tamanho corporal do feto e o potencial materno para o fornecimento de nutrientes influenciariam os crescimentos pré e pós-natal, resultando em inadequado atendimento das exigências nutricionais de bezerros machos produtos do cruzamento de raças pesadas com vacas Nelore.

Contrastes ortogonais (Tabela 4) entre Nelore e os demais grupos e entre RN com MN são instrumen-

Tabela 3 - Médias dos quadrados mínimos e respectivos erros-padrão de características ponderais em bovinos Nelore e cruzas com Nelore, para machos e fêmeas, em Paranavaí-PR

Table 3 - Least squares means and respective standard errors of growth traits in crossbred and purebred Nelore cattle, for males and females, in Paranavaí-PR

\begin{tabular}{|c|c|c|c|}
\hline \multirow[t]{2}{*}{$\begin{array}{l}\text { Grupo genético } \\
\text { Genetic group }\end{array}$} & \multicolumn{3}{|c|}{$\begin{array}{l}\text { Médias dos quadrados mínimos e erros-padrão (kg) } \\
\text { Least square means and standard errors }\end{array}$} \\
\hline & $\mathrm{PNT}^{1}$ & $\mathrm{PDS}^{1}$ & GMD_ND ${ }^{1}$ \\
\hline$\overline{\text { Nelore }(\mathrm{N})-\mathrm{M}^{2}}$ & $29,8 \pm 0,46$ & $148,9 \pm 1,8$ & $0,537 \pm 0,009$ \\
\hline Nelore $(\mathrm{N})-\mathrm{F}^{3}$ & $27,2 \pm 0,43$ & $133,7 \pm 1,7$ & $0,482 \pm 0,008$ \\
\hline Guzerá x N - M & $30,1 \pm 0,53$ & $154,0 \pm 1,9$ & $0,565 \pm 0,009$ \\
\hline Guzerá x N - F & $27,9 \pm 0,53$ & $141,2 \pm 2,0$ & $0,513 \pm 0,009$ \\
\hline Red Angus x N - M & $29,7 \pm 0,54$ & $172,1 \pm 2,1$ & $0,645 \pm 0,010$ \\
\hline Red Angus x N - F & $29,1 \pm 0,52$ & $162,9 \pm 2,0$ & $0,609 \pm 0,009$ \\
\hline Marchigiana x N-M & $31,0 \pm 0,53$ & $163,6 \pm 2,1$ & $0,588 \pm 0,010$ \\
\hline Marchigiana x N - F & $31,5 \pm 0,59$ & $160,5 \pm 2,4$ & $0,579 \pm 0,011$ \\
\hline
\end{tabular}




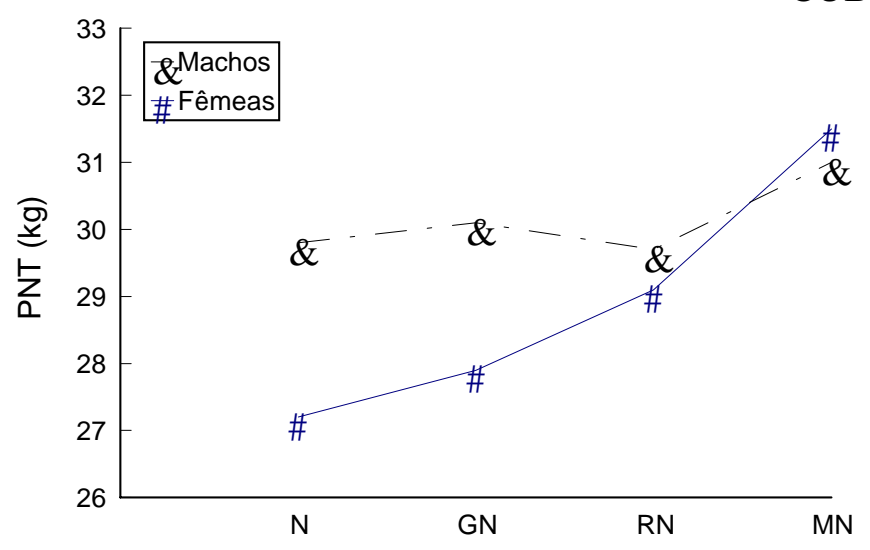

Grupo genético Genetic group

Figura 1- Pesos ao nascer dos bezerros (PNT), de acordo com o sexo e o grupo genético, em que $\mathrm{N}=$ Nelore, $\mathrm{GN}=$ Guzerá $\times$ Nelore, $\mathrm{RN}=$ Red Angus $\times$ Nelore e $\mathrm{MN}=$ Marchigiana $\times$ Nelore.

Figure 1 - Calves birth weights (PNT), according to genetic group and sex, where $N=$ Nellore, GN = Guzera $\times$ Nellore, $R N=$ Red Angus $x$ Nellore e $M N=$ Marchigiana $\times$ Nellore .

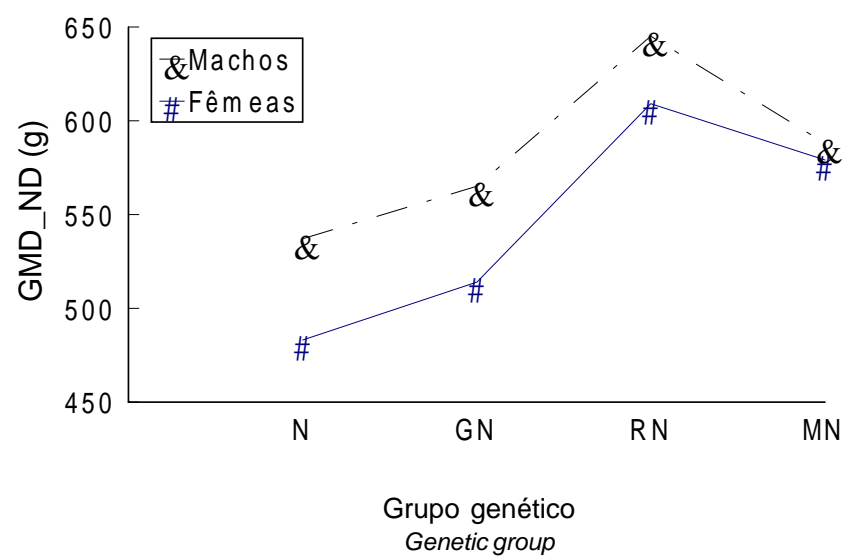

Figura 3 - Ganho médio diário de peso do nascimento à desmama dos bezerros (GMD ND), de acordo com o sexo e o grupo genético, em que $\mathrm{N}=$ Nelore, $\mathrm{GN}=$ Guzerá $\times$ Nelore, $\mathrm{RN}=$ Red Angus $\times$ Nelore e $\mathrm{MN}=$ Marchigiana $\times$ Nelore.

Figure 3 - Calves average daily gain of weight (GMD ND), according to genetic group and sex, where $N=$ Nellore, GN $=$ Guzera $\times$ Nellore, $R N=$ Red Angus $x$ Nellore e $M N=$ Marchigiana $\times$ Nellore.

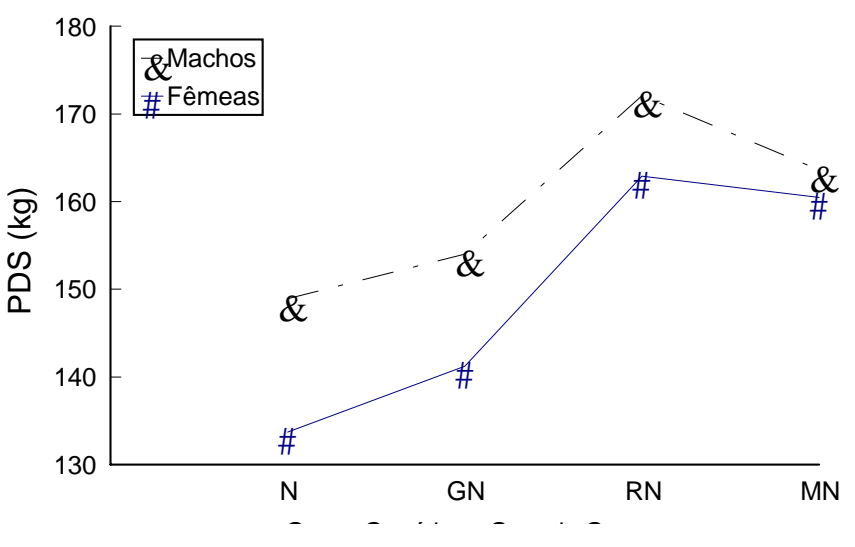

Grupo genético Genetic group

Figura 2 - Pesos à desmama (PDS), de acordo com o sexo e o grupo genético, em que $\mathrm{N}=$ Nelore, $\mathrm{GN}=$ Guzerá $\times$ Nelore, $\mathrm{RN}=$ Red Angus $\times$ Nelore e $\mathrm{MN}=$ Marchigiana $\times$ Nelore.

Figure 2 - Weaning weights (PDS), according to genetic group and sex, where $N=$ Nellore, GN = Guzera $\times$ Nellore, $R N=$ Red Angus $\times$ Nellore e MN = Marchigiana $\times$ Nellore

tos úteis para melhor entendimento das diferenças entre grupos genéticos. Os resultados apresentados na Tabela 4 permitem a predição do comportamento futuro das raças envolvidas. Embora não sejam esperadas diferenças entre os pesos ao nascer de bezerros Nelore e GN, tem-se a expectativa de que os bezerros filhos de touros Guzerá com vacas Nelore pesem ao redor de $6,3 \mathrm{~kg}$ a mais na desmama e tenham ganho médio diário de aproximadamente $30 \mathrm{~g}$ a mais que as progênies de touros Nelore no período prédesmama. Os bezerros RN não deverão pesar mais que os Nelore quando do nascimento, mas deverão pesar 26,2 kg a mais na desmama e ter um GMD_ND de aproximadamente $117 \mathrm{~g}$ a mais na fase que antecede a desmama. Já os bezerros MN deverão pesar aproximadamente $2,8 \mathrm{~kg}$ a mais ao nascer, $20,8 \mathrm{~kg}$ a mais na desmama, e ter GMD_ND de 74 g superior, em relação aos Nelore. Embora os bezerros $\mathrm{MN}$ venham a nascer com $1,9 \mathrm{~kg}$ a mais que os $\mathrm{RN}$, devem atingir a desmama pesando $5,5 \mathrm{~kg}$ a menos, com ganho diário pré-desmama de $43 \mathrm{~g}$ menor. 
Tabela 4 - Estimativas de contrastes ortogonais e respectivos erros-padrão em bovinos Nelore e cruzas com Nelore, em Paranavaí-PR

Table 4 - Estimates of orthogonal contrasts and respective standard errors in crossbred and purebred Nelore beef cattle, in Paranavaí-PR

\begin{tabular}{|c|c|c|c|}
\hline \multirow[t]{2}{*}{$\begin{array}{l}\text { Contraste } \\
\text { Contrast }\end{array}$} & \multicolumn{3}{|c|}{$\begin{array}{l}\text { Estimativas e respectivos erros-padrão } \\
\text { Estimates and respective standard errors }\end{array}$} \\
\hline & $\mathrm{PNT}^{1}$ & $\mathrm{PDS}^{1}$ & GMD_ND $^{1}$ \\
\hline $\begin{array}{l}\mathrm{N}^{2}-\text { Guzerá x Nelore } \\
\mathrm{N}^{2}-\text { Red Angus x Nelore } \\
\mathrm{N}^{2}-\text { Marchigiana x Nelore } \\
\mathrm{RN}-\mathrm{MN}^{2}\end{array}$ & $\begin{array}{l}-0,513 \pm 0,503 \mathrm{~ns} \\
-0,895 \pm 0,525 \mathrm{~ns} \\
-2,773 \pm 0,551 * * * \\
-1,878 \pm 0,612 * * *\end{array}$ & $\begin{array}{c}-6,296 \pm 1,88 * * * \\
-26,209 \pm 1,99 * * * \\
-20,756 \pm 2,17 * * * \\
5,453 \pm 2,37 * *\end{array}$ & $\begin{array}{c}-0,030 \pm 0,009 * * * \\
-0,117 \pm 0,010 * * * \\
-0,074 \pm 0,010 * * * \\
0,043 \pm 0,011 * * *\end{array}$ \\
\hline
\end{tabular}

1 PNT = peso ao nascimento; PDS = peso à desmama; GMD_ND = ganho médio diário de peso do nascimento à desmama; ns = não-significativo; ${ }^{* *}=\mathrm{P}<0,05 ;{ }^{* * *}=\mathrm{P}<0,01$.

$2 \mathrm{~N}=$ Nelore, $\mathrm{RN}-\mathrm{MN}=$ Red Angus $\times$ Nelore - Marchigiana $\times$ Nelore.

$1 P N T=$ calf birth weight; $P D S=$ calf weaning weight; GMD_ND = average daily gain from birth to weaning; $n s=$ non significant; ${ }^{* *}=P<.05 ;{ }^{* * *}=P<.01$.

${ }^{2} N=$ Nellore; $R N-M N=$ Red Angus $x$ Nellore - Marchigiana $\times$ Nellore.

\section{Conclusões}

Entre os efeitos fixos importantes atuando sobre características pré-desmama de bovinos Nelore e cruzamentos com Nelore, vale destacar o efeito de mês de nascimento sobre o ganho diário do nascimento à desmama e sobre o peso à desmama, pois a época de nascimentos pode ser planejada pelo homem para seu benefício. A estação de nascimentos de primavera/verão produziu melhores ganhos e pesos que a de outono e, dentro da primeira, os melhores resultados foram obtidos com nascimentos no início, principalmente nos meses de julho e agosto, o que indica que, quanto mais cedo se iniciar a estação de monta de primavera/verão, maiores serão os benefícios esperados para o desempenho pré-desmama.

O efeito fixo de grupo genético indica melhor desempenho pré-desmama para o grupo Red Angus x Nelore, seguido de Marchigiana x Nelore e Guzerá $x$ Nelore, indicativo de que cruzamentos de raças de corte com matrizes Nelore são opções disponíveis para os criadores para melhorar o desempenho prédesmama de seus rebanhos.

A importante interação grupo genético x sexo do bezerro, com a diferença a favor dos machos diminuindo sistematicamente nos grupos Red Angus x Nelore e Marchigiana x Nelore, indica que possivelmente as condições ambientais em que os animais experimentais foram produzidos limitaram o pleno desenvolvimento do potencial de crescimento dos bezerros machos nos referidos grupos e, em condições ideais de pastagens, este potencial pode se manifestar por inteiro.

\section{Referências Bibliográficas}

ALENCAR, M.M., BARBOSA, P.F., TULLIO, R.R. et al. 1995. Peso à desmama de bezerros da raça Nelore e cruzados Canchim x Nelore e Marchigiana x Nelore. R. Soc. Bras. Zootec., 24(6):917-925.

ALENCAR, M.M., TREMATORE, R.L., BARBOSA, P.F. et al. 1997. Desempenho de bezerros filhos de touros das raças Nelore e Canchim e cruzados (F1) Charolês x Nelore e Piemontês x Nelore. R. Bras. Zootec., 26(3):461-466.

ALENCAR, M.M., TREMATORE, R.L., OLIVEIRA, J.A. et al. 1998. Características de crescimento até a desmama de bovinos da raça Nelore e cruzados Charolês x Nelore. R. Bras. Zootec., 27(1):40-46.

BERGER, P.J., CUBAS, A.C., KOEHLER, K.J. et al. 1992. Factors affecting dystocia and early calf mortality in Angus cows and heifers. J. Anim. Sci., 70:1775-1786.

CAMPOS, L.T., SILVA, P.R., FRIES, L.A. 1989. Fatores de correção para efeitos ambientais que afetam o ganho do nascimento à desmama em bovinos da Raça Nelore. In: CONGRESSO BRASILEIRO DE PESQUISA DE ZEBU, 1, Uberaba, 1988. Anais... Uberaba: EPAMIG CRTP, 1989. p.108-123.

CARTWRIGHT, T.C. 1970. Selection criteria for beef cattle for the future. J. Anim. Sci., 30:706-711.

CUBAS, A.C., BERGER P. J., HEALEY, M. H. 1991. Genetic parameters for calving ease and survival at birth in Angus field data. J. Anim. Sci., 69:3952-3958.

EUCLIDES FILHO, K., FIGUEIREDO, G.R., DA SILVA, L.O. et al. Pesos ao nascer e à desmama e ganho pré-desmama de Nelore e seus mestiços com Fleckvieh, Chianina, Charolês e Angus. In: REUNIÃO ANUAL DA SOCIEDADE BRASILEIRA DE ZOOTECNIA, 33, 1996. Fortaleza. Anais... Fortaleza: SBZ, 1996, v.1, p.164-166.

EUCLIDES FILHO, K., FIGUEIREDO, G.R., DA SILVA, L.O. et al. 1998. Idade aos $165 \mathrm{~kg}$ de peso vivo para progênies de Nelore, Fleckvieh, Chianina, Charolês, F1's e retrocruzas. R. Bras. Zootec., 27(5):899-905.

FITZHUGH JR., H.A., TAYLOR, S.C.S. 1971. Genetic analysis of degree of maturity. J. Anim. Sci., 33:717-725.

GALlANT, A.R., FULLER, W.A. 1973. Fitting segmented polynomial regression models whose join points have to be estimated. J. Amer. Stat. Assoc., 68:144-147. 
GREGORY, K.E. 1961. Improvement of beef cattle through breeding methods. Research Bulletin, North Central Region Publication, RRS/USDA/USA, n.196, 120p.

IAPAR. Cartas climáticas do Estado do Paraná. 1974. Londrina: Instituto Agronômico do Paraná. 49p (Documento 18).

KOCH, R.M., DICKERSON, G.E., CUNDIFF, L.V. et al. 1985. Heterosis retained in advanced generations of crosses among Angus and Hereford cattle. J. Anim. Sci., 60:1117-1132.

KOLB, E.L. Fisiologia veterinária. 1984. Rio de Janeiro: Guanabara Koogan S.A., 4.ed. 612p.

MAZZA, M.C. Estudo das influências sobre o crescimento até um ano de idade em um rebanho Guzerá. Belo Horizonte/ MG: UFMG. 44p. 1984. Dissertação (Mestrado em Zootecnia) - Universidade Federal de Minas Gerais, 1984.

MINISTÉRIO DA AGRICULTURA. 1970. Levantamentos de reconhecimento dos solos do noroeste do Estado do Paraná. Rio de Janeiro: Ministério da Agricultura. 102p (Boletim Técnico 14).

MUNIZ, C.A.S.D., QUEIROZ, S.A. 1998. Avaliação do peso à desmama e do ganho médio de peso de bezerros cruzados, no Estado do Mato Grosso do Sul. R. Bras. Zootec., 27(3):504-512.
PEROTTO, D., CUBAS, A.C., MOLETTA, J.L. et al. 1998. Pesos ao nascimento e à desmama e ganho de peso do nascimento à desmama de bovinos Charolês, Caracu e cruzamentos recíprocos. R. Bras. Zootec., 27(4):730-737.

PEROTTO, D., ABRAHÃO, J.J.S., CUBAS, A.C. 1999. Efeitos da raça e da heterozigose sobre características ponderais de bezerros Nelore e mestiços Red Angus x Nelore. R. Bras. Zootec., 28(3):504-511.

PRESTON, T.R., WILLIS, M.W. 1974. Intensive beefproduction. 2.ed., Oxford: Pergamon Press. 567p.

SAS INSTITUTE INC. 1985. SAS User's guide: statistics. 5.ed., Cary, NC: SAS Inst. Inc. 956p.

Recebido em: 08/02/00

Aceito em: 24/01/01 